\title{
Real-Time Mobile Communication of Power Requirements for Electric Vehicles
}

\author{
Derek Smith
}

\author{
"Adding an electric vehicle or two to a neighbourhood"s \\ can be like adding another house, and it can stress \\ the equipment that services those houses. We're \\ talking about doubling the load of a conventional \\ home. It's big. \\ Karl Rábago \\ Vice President of Distributed Energy Services \\ Austin Energy \\ (tinyurl.com/ad9xksd)
}

\begin{abstract}
The periodic power requirements of an electric vehicle are difficult to predict because the vehicle's location, the amount of charge remaining in its batteries, and the timing of its next charge are not known. For clusters of electric vehicles, the problem is magnified, and there is a risk that the demand will strain and overload a power utility's infrastructure. Operational managers are left with reactive management of the infrastructure that may defer or prevent a vehicle charge to balance power demands and safeguard the infrastructure.

In this article, the following key concepts are analyzed to provide background on the problem and to outline the requirements of any solution: i) demand uncertainty and reactive management approaches, ii) electric vehicle power requirements, and iii) demand-management telecommunication capabilities. Then, by abstraction, induction, and creative synthesis, a novel solution to the problem is proposed to provide real-time mobile communication of power requirements. The proposed solution has potential to create new service and business opportunities to managers and entrepreneurs.
\end{abstract}

\section{Introduction}

As indicated in the opening quotation, the power requirements of an electric vehicle are comparable to a house. As adoption of electric vehicles increases, the increased and variable demand will be akin to squeezing an unknown number of new houses and their power requirements onto a neighbourhood's existing power grid. But these new "houses" bring additional variables and challenges. Consider a dynamic neighbourhood of mobile "houses" that are capable of roaming the city and taking their power demands with them on the road to new and unknown places.

These electric vehicles create an opportunity to fundamentally change the manner in which electric utilities manage their infrastructure and do business with their customers. It is paramount for managers at the electrical utilities to transform from reactive management of their infrastructure to proactive management in order to avoid potential strain or damage to the system from the significantly increasing number and potential clustering of electric vehicles at unknown locations in the utility infrastructure.

Accurately locating, predicting, and communicating the amount of energy required at any given time for periodically charging electric vehicles is a challenge. As a consequence, should the current approach of reactive management of the infrastructure continue, it will become highly problematic as the number of electric vehicles increase and, correspondingly, the peak power demands increase.

Previous research proposes a number of different reactive approaches to avoid these power demands caused 


\section{Real-Time Mobile Communication of Power Requirements for Electric Vehicles}

\section{Derek Smith}

by electric vehicles. For example, operational managers can react to the pending imbalance by: delaying or postponing access, prioritizing access, restricting time access, or buffering energy. However, real-time determination of power requirements and proactive management of the infrastructure have not been considered by previous researchers. Furthermore, realtime mobile monitoring of electric vehicles and communication to enable proactive management has not been considered.

The contributions of this article are:

1. Identification and grouping of key elements for electric vehicle power demand

2. Identification of key telecommunication technologies to solve the problem

3. A proposed solution based upon an abstraction of the problem

4. A description of a potential minimal viable product for real-time mobile communication of vehicle location and power requirements to service providers or electric utilities

In the next section, key concepts necessary to understand the problem and the requirements of any solution are identified. Next, a proposed solution is inductively synthesized from an abstraction of the problem including identification and grouping of mobile data and enabling telecommunication technologies. Then, the proposed solution is further synthesized as a minimal viable product for the real-world problem. Finally, a conclusion is provided.

\section{Key Concepts}

This section identifies important factors that contribute to the problem of managing the power requirements of electric vehicles, including demand uncertainty elements and reactive management approaches to balance the supply and demand of electricity, sources of power uncertainty in predicting electric vehicle power requirements, and demand-management telecommunication capabilities.

\section{Demand uncertainty and reactive demand-management} approaches

Based on a review of the literature, Mullan and colleagues (2012; tinyurl.com/ahej3ty) identified the impacts on electricity grids of large-scale recharging of electric vehicles as one of "the main policy issues associated with the electrification of the vehicle fleet". These impacts arise, in part, because of the management challenges posed by two types of uncertainty: key demand uncertainty and key imbalance uncertainty. A key demand uncertainty is the amount of power required for a charge for an uncertain duration of the charge (Masoum et al., 2011; tinyurl.com/beu7164), and this will impact the ability to manage power demands. This impact will increase as the number of electric vehicles increases or cluster together in a particular area of a utility's infrastructure. A key imbalance uncertainty is the location of electric vehicles in neighbourhoods or city parking lots, and it may also cause an unmanageable demand imbalance in particular areas (Kang and Recker, 2009; tinyurl.com/bhgwuh2). Neighbourhoods and city parking lots are two examples of areas where clustering will occur, but the timing and intervals of demand are unknown.

The current approaches to reduce the impact on electricity grids and ensure a balance of electricity are limited to reactive response-management techniques and include:

- turning off electrical devices during peak powerdemand periods

- offering different time slots after peak power-demand hours (Kang and Recker, 2009; tinyurl.com/bhgwuh2)

- restricting access to defined windows of time or other scheduling approaches

- delaying access

- utilizing on-time scheduling of power demand (Koutsopoulos and Tassiulas, 2011; tinyurl.com/d5q4vc2)

This analysis suggests that, as we increase new demand uncertainties and new imbalance uncertainties, the only approach for operational managers is a form of reactive management wherein charging of the electric vehicle will be delayed, restricted, or unavailable without any capability for planning the generation of electricity.

\section{Electric vehicle power requirements}

There are a number of different types of electric vehicles available on the market today. For example, the Mitsubishi Imiev (tinyurl.com/cklby2q) is an electric 


\section{Real-Time Mobile Communication of Power Requirements for Electric Vehicles}

\section{Derek Smith}

city-commuter car with a driving range of $155 \mathrm{~km}$ on a single charge. The Nissan Leaf is also an electric citycommuter car, and it has a range of $160 \mathrm{~km}$ on a single charge (tinyurl.com/d9mj217). The time to charge these vehicles varies and is dependent upon the charge rate. It may require as much as 18 hours or as little as 26 minutes for the batteries to reach $80 \%$ capacity. The Tesla Model S (tinyurl.com/2azpfue) is an electric vehicle designed with a sports car aesthetic and handling, and it includes an optional high-performance 416hp electric motor. With a charge that provides $100 \mathrm{~km}$ of distance per hour, the vehicle can travel up to $480 \mathrm{~km}$ on a single charge.

Given the different designs and uses of electric vehicles, it is not surprising that different types of electric vehicle have different power requirements (Weiller, 2011; tinyurl.com/b2hlxmk). Plug-in hybrid electric vehicles that have an auxiliary, fuel-based engine may continue to operate when the battery charge is low, but plug-in electric vehicles may not. Kang and Recker (2009; tinyurl.com/bhgwuh2) point out that electric commuter vehicles and electric sports cars have different ranges, and that battery capacity will also affect power requirements. Also, the type of charge (i.e., slow, normal, or fast) affects the peak power demand for electricity (Fernández et al., 2011: tinyurl.com/aoape28; Weiller, 2011: tinyurl.com/b2hlxmk).

Driving patterns and timing also affect power requirements. The effects of driving patterns are random both from the perspective of departure and arrival (Weiller, 2011: tinyurl.com/b2hlxmk; Masoum et al., 2011: tinyurl.com/beu7l64) as well as the length of each trip. The power requirements are similarly affected by timing, which includes the amount of time required to provide the power to the electric vehicle, the type of day (Weiller, 2011; tinyurl.com/b2hlxmk), and the time of day (Fernández et al., 2011: tinyurl.com/aoape28).

Geographic location (Weiller, 2011; tinyurl.com/b2hlxmk) and the season (Clement-Nyns et al., 2010; tinyurl.com/ b5grkgu) will also affect requirements. For example, battery efficiency will be lower in colder weather and power consumption will be greater in mountainous areas.

This analysis suggests that the type of vehicle technology, range, battery capacity, type of change, differing patterns of use, and geographic location compound the difficulty in predicting electric vehicle power requirements. These factors will make it more difficult for oper- ational managers to manage peak demands and plan peak electrical needs due to the uncertainty of these requirements.

Demand-management telecommunication capabilities Telecommunication capabilities exist between a utility and a customer's smart meter that provide a limited and very basic form of peak-load management (Mullan et al., 2012; tinyurl.com/ahej3ty). The capabilities provided by smart meters allow the utility to control a select set of non-critical electrical devices for demand power management (Koutsopoulos and Tassiulas, 2011; tinyurl.com/d5q4vc2). The utility may coordinate peakload management by selecting a partial load for an electrical device, delaying an electrical device for a specified period of time, or reducing a load for an electrical device (Shahnia et al., 2012; tinyurl.com/bzylua2). An electric vehicle plugged into a particular circuit at a customer's premises could also be controlled this way as part of peak-load management.

A further extension of this concept applies directly to chargers located in electric vehicles. This extension provides response management when an electric vehicle is being charged away from a customer's premises, such as, at a municipal charging station (Masoum et al., 2011; tinyurl.com/beu7164). In this situation, telecommunication capabilities would allow the utility to permit or possibly delay charging of an electric vehicle. Commuter versions of electric vehicles have a small range and typically require frequent charging. A consumer's expectation would be that, if the vehicle is plugged in, then it should be able to receive a charge without delay. To either fully charge or to top up a charge to continue on the next portion of the owner's journey is acceptable, but a long unknown delay in charging an electric vehicle will not be acceptable.

Even though there are some telecommunication capabilities in place already, these capabilities are used for reactive response management to reduce a customer's demand for electricity during peak-demand periods and allow the customer's demand during off-peak periods.

This analysis suggests that telecommunications between a utility and the customer's device is available through a smart meter or electric vehicle charger for response demand management. However, response demand management is limited to reducing load during peak demands or scheduling load to off-peak demand 


\section{Real-Time Mobile Communication of Power Requirements for Electric Vehicles}

\section{Derek Smith}

periods. There is no capability to determine the amount of load required, the duration of the load, the timing of the load, or the location of a load for a mobile device.

\section{Proposed Solution}

In this section, a proposed solution to the problem of managing the periodic power requirements of electric vehicles is described through an abstraction of the problem, as illustrated in Figure 1. This abstraction was created by identifying and grouping mobile data into key categories, identifying enabling telecommunication technologies, and applying an inductive approach and creative synthesis to propose the solution.

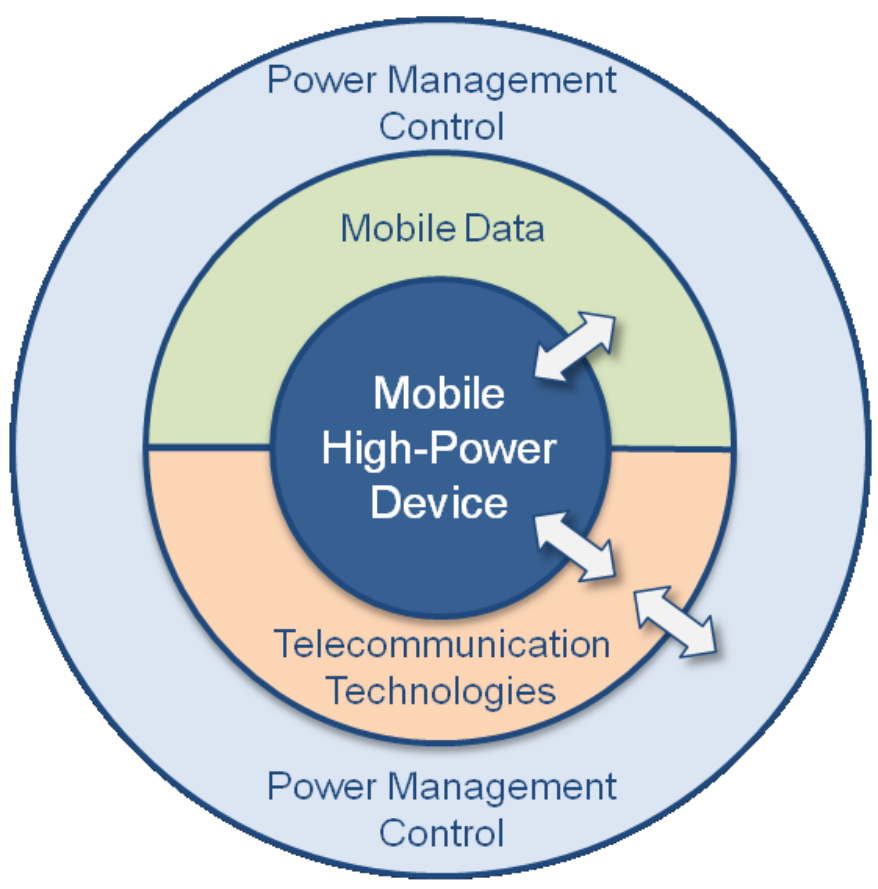

Figure 1. Abstraction

The proposed solution will allow utilities to locate, track, and identify in real time the power requirements of mobile devices with high power requirements. The solution enables proactive power management and control for individual, mobile high-power devices and clusters of such devices. The central construct is a mobile high-power device, which could be any electric device that requires power. The device is mobile, or movable, meaning that it can be transported from location to location. Hence, its location varies within the electrical utility's infrastructure.
The mobile data construct is related to the mobile highpower device construct in that it contains real-time mobile data relevant to the device that must be determined in real time and is grouped into two broad categories of information: charge requirements and device location.

The telecommunication technologies construct includes technology that provides for communication capability as well as device monitoring. The relationship to the mobile high-power device is through monitoring and logging mobile data. The relationship to the power management control construct is from communicating the mobile data for the device to the power management control construct as well as communicating commands from the power management control construct to the device. The power management control construct includes technology that manages the supply and demand of electric power in the utility's infrastructure.

Previous research has identified existing technologies with the capability of supporting reactive demand management and control of power-consuming devices through smart meters and electric vehicle chargers. In this scenario, a signal is sent to activate or deactivate a power-consuming device, thus enabling a limited form of reactive demand management. When demand exceeds the supply, the power-consuming devices may be deactivated for a period of time and then reactivated when supply is available. In the next section, additional enabling telecommunication technologies for the proposed solution are identified through personal experience and an Internet search.

\section{Enabling telecommunication technologies}

Data loggers are electronic devices that capture realtime data including operational parameters. Data loggers may interface with a bus that is located with the mobile high-power device and may be programmed to monitor and $\log$ mobile data. In the case of a mobile high-power device, the data logger monitors and captures the first broad category of information for charge requirements.

Global positioning devices are also available. These electronic devices provide, as a minimum, location data. They may also provide other types of data including speed and direction. The global positioning device, or the data logger coupled to the global positioning device, monitors and logs the second broad category of information to determine location of the mobile highpower device. 


\section{Real-Time Mobile Communication of Power Requirements for Electric Vehicles}

\section{Derek Smith}

Data communication devices provide data communication capability and generally include cellular, WiFi, Bluetooth, and satellite communication technologies. Coupling a data logger with a global positioning device and a data communications device with a mobile highpower device provides the basic capability to send mobile data and receive commands over a telecommunication system to a remote site.

The proposed solution is therefore a system. A mobile component of the system includes telecommunication technology for monitoring and logging mobile data relevant to a mobile high-power device. This includes a data logger, a global positioning device, and a data communication device for sending and receiving data. A remote part of the system includes a power management control component located at a remote site with the capability to receive mobile data for storing on a serverbased system for use by existing demand-management technology. By knowing the location of mobile highpower devices within the utility infrastructure (e.g., a specific section of the grid) and the corresponding locations of other mobile high-power devices, as well as their electric power requirements, the service provider may apply proactive power management control and distribution to supply and balance power to the mobile high-power devices. Commands to start or stop consumption of power may be sent over the telecommunication's existing network to the mobile high-power device in order to provide access to power or restrict access to power. This system provides a timely, distributed supply of energy to the mobile high-power devices, and even clusters of these devices, by proactively controlling power management without risk to the utility infrastructure or interruption to a customer's supply of electric power.

\section{Minimum Viable Product}

This section provides a more detailed description of the synthesis between the proposed solution and the abstract problem. Here, the solution is portrayed as a minimum viable product (tinyurl.com/yhstpma) and is applied to the real-world scenario of electric vehicles operating within a utility infrastructure.

In this scenario, the mobile high-power device is a electric vehicle in the real world. The power category of mobile data maps parameters identified in the literature, such as battery capacity, amount depleted, power required for a full charge, power required for next nonfull charge, and vehicle data (e.g., vehicle identification number, plug-in hybrid vehicle, plug-in electric vehicle, battery type). The location category of mobile data maps parameters identified in the literature, such as geographic location and landscape conditions (e.g., mountainous area, city, countryside). A trip category of data is also introduced that can be determined from both the vehicle category of mobile data and the location category of mobile data.

The data logger is a vehicle data logger that interfaces to the vehicle through an on-board diagnostics (OBD II) port in the vehicle and the vehicle controller area network (CAN II) data bus. The data logger monitors, captures, and logs the power category of mobile data. The data logger may also send commands over the vehicle CAN II data bus to control subsystems in the vehicle. For example, it is capable of controlling (i.e., activating or deactivating) an electric vehicle charger. A global positioning system is coupled to the data logger and monitors, captures, and logs the location category of mobile data. A communication device may also be coupled to the data logger and the global positioning system to send mobile data over a telecommunications network and receive commands for the data logger. The data logger, global positioning system, and communications device enable the electric vehicle to monitor, log, and send mobile data to a remote site and receive commands from the remote site.

At the remote site, the mobile data for an electric vehicle is stored on a remote server and monitored in real time. The trip category of data is determined by assessing both the power category of mobile data and the location category of mobile data. This provides information on driving patterns, future charge requirements for the plug-in electric vehicle, and location information as it relates to the infrastructure and other electric vehicles with the aim of identifying potential cluster problems. Essentially, this system provides the capability to determine the real-time amount of demand load and timing of the load for better planning of both power generation and demand management. The system includes a new capability for off-loading an excess supply of power to electric vehicles waiting to receive power.

When charging is required for an electric vehicle, a request is sent over the communication device to the remote site. The remote site determines the real-time amount of power required to charge the electric vehicle and schedules charging with the demand-management system based on peak-demand loads, surplus power, or degree of clustering. Then, based on the schedule, a "start charge" command is sent to the communication 


\section{Real-Time Mobile Communication of Power Requirements for Electric Vehicles}

\section{Derek Smith}

device and the data logger activates the electric vehicle charger. If required, a "stop charge" command could also be sent to the communication device and the data logger would then deactivate the electric vehicle charger. The process repeats for all plug-in electric vehicles with an installed minimum viable product.

This minimum viable product description illustrates that charging of electric vehicles, including clusters of vehicles, is possible without risk to the utility's infrastructure.

\section{Conclusion}

The implications of real-time monitoring of electric vehicles and communication of location and power requirements to service providers and utilities are twofold. First, it permits a change from reactive to proactive management of the electrical infrastructure. Second, it solves the problem of clustering and has the potential to prevent significant risk to the infrastructure associated with clustering of electric vehicles. This solution results in better peak-load management and the creation of oversupply safeguard capabilities.

The proposed solution allows electric utility managers and executives to be equipped with better power-supply planning and to permit proactive demand management. As well, the solution provides entrepreneurs with a potential new business opportunity and a minimum viable product opportunity for deployment to early adopters.

More broadly, real-time monitoring of mobile highpower devices and communication of location and power requirements is applicable to other mobile intelligent power-consuming devices in the home, hospitals, construction sites, airports, and a variety of other commercial and manufacturing applications. Given the unpredictable nature and growing worldwide demand for power, this approach can provide value on a global scale.

\section{About the Author}

Derek Smith is the Founder and Principal of Magneto Innovention Management, an Intellectual Property consulting firm that assists entrepreneurs and small businesses to navigate and grow their international patent portfolios. Derek has over 20 years of experience working as an intellectual property management consultant and patent agent for IBM Canada, Bell Canada and, most recently, Husky Injection Molding Systems where he was Director, Global Intellectual Property. His role at Husky included working with international counsel to resolve oppositions before the European Patent Office and the United States Patent and Trademark Office. Prior to entering the field of IP, Derek was an advisory engineer at IBM Canada where he was involved in a variety of leading-edge software development projects. Derek is currently a graduate student in the Technology Innovation Management program at Carleton University in Ottawa, Canada. He also holds a BEng degree in Systems and Computer Engineering from Carleton University and is a registered patent agent in both Canada and the United States.

Citation: Smith, D. 2012. Real-Time Mobile

Communication of Power Requirements for Electric

Vehicles. Technology Innovation Management Review.

December 2012: 22-27. 